\title{
Development of minimal-change glomerular disease and Hashimoto's thyroiditis during the treatment of sarcoidosis
}

\author{
Fumiaki Ando • Tomokazu Okado • \\ Eisei Sohara - Tatemitsu Rai • \\ Shinichi Uchida $\cdot$ Sei Sasaki
}

Received: 15 November 2012/ Accepted: 25 February 2013/Published online: 17 March 2013

(C) Japanese Society of Nephrology 2013

\begin{abstract}
Minimal-change glomerular disease, sarcoidosis and autoimmune thyroid disease rarely occur in the same patient. We herein report a patient in which minimal-change glomerular disease and Hashimoto's thyroiditis developed during the treatment of sarcoidosis with steroids. A 66-yearold female was admitted to our hospital with symptoms of nephrotic syndrome. Nine months before admission, she was diagnosed as having ocular and pulmonary sarcoidosis, for which prednisolone at an initial dose of $40 \mathrm{mg}$ /day was started. When the dose of prednisolone was tapered to $20 \mathrm{mg} /$ day, she noticed swelling of the lower extremities. Examination confirmed the simultaneous occurrence of minimal-change glomerular disease and Hashimoto's thyroiditis, which were diagnosed based on kidney histology, ultrasonography of the thyroid gland and positive antithyroglobulin antibodies. We used intravenous methylprednisolone pulse therapy followed by $40 \mathrm{mg} /$ day oral prednisolone. The patient achieved complete remission of nephrotic syndrome and steroids were tapered without relapse.
\end{abstract}

Keywords Minimal-change glomerular disease .

Sarcoidosis · Hashimoto's thyroiditis

\section{Introduction}

The occurrence of nephrotic syndrome in patients with autoimmune thyroid disease or sarcoidosis has been

F. Ando · T. Okado $(\bowtie) \cdot$ E. Sohara $\cdot$ T. Rai $\cdot$ S. Uchida .

S. Sasaki

Department of Nephrology, Tokyo Medical and Dental

University, 1-5-45 Yushima, Bunkyo-ku,

Tokyo 113-8510, Japan

e-mail: tokado.kid@tmd.ac.jp reported. Pathologically, membranous glomerulonephritis is more frequent, and minimal-change glomerular disease (MCD) is relatively rare in both sarcoidosis and autoimmune thyroid disease $[1,2]$. Only a few cases have been reported in which $\mathrm{MCD}$, sarcoidosis and autoimmune thyroid disease occurred in the same patient. Such reports suggest the presence of immunological links between these three conditions.

We herein describe a case in which sarcoidosis occurred first, followed by a diagnosis of both MCD and Hashimoto's thyroiditis after 3 months of treatment with prednisolone.

\section{Case report}

A 66-year-old female was admitted to our hospital with nephrotic syndrome. Nine months prior to admission, she visited another hospital with symptoms of blurred vision, and ocular sarcoidosis was diagnosed based on ophthalmologic examination. Chest X-ray and computed tomography (CT) scan confirmed the presence of bilateral lymphadenopathy (Fig. 1). Gallium-67 citrate scintigram showed increased uptake in the pulmonary hilum and mediastinum, but renal uptake was not present (Fig. 2). Serum angiotensin-converting enzyme (ACE) levels were not elevated. Six months later, prednisolone at an initial dose of $40 \mathrm{mg} /$ day was started by her previous doctor for the treatment of ocular sarcoidosis. When the dose of prednisolone was tapered to $20 \mathrm{mg} / \mathrm{day}$, she noticed swelling of the lower extremities.

On admission, physical examination revealed pitting oedema below the knees. Blood pressure was 181/94 mmHg. Respiratory, cardiovascular, neurological and skin examination was normal. The thyroid gland was unremarkable. 


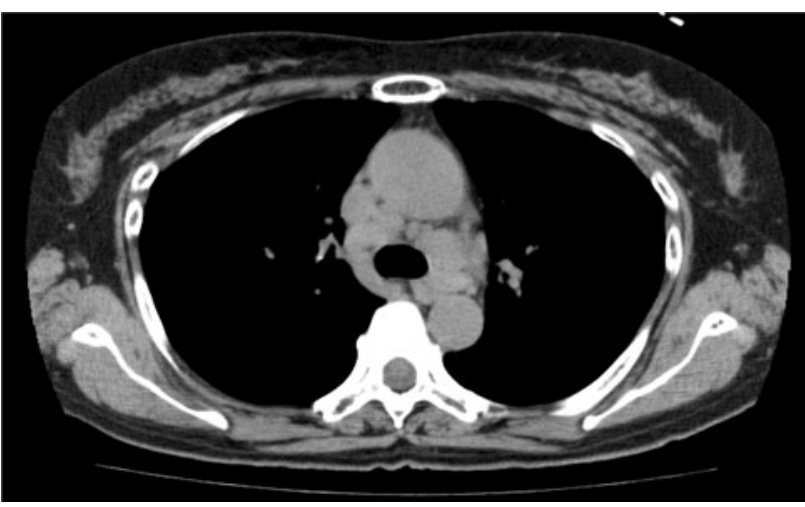

Fig. 1 Computed tomography (CT) scan confirming bilateral lymphadenopathy

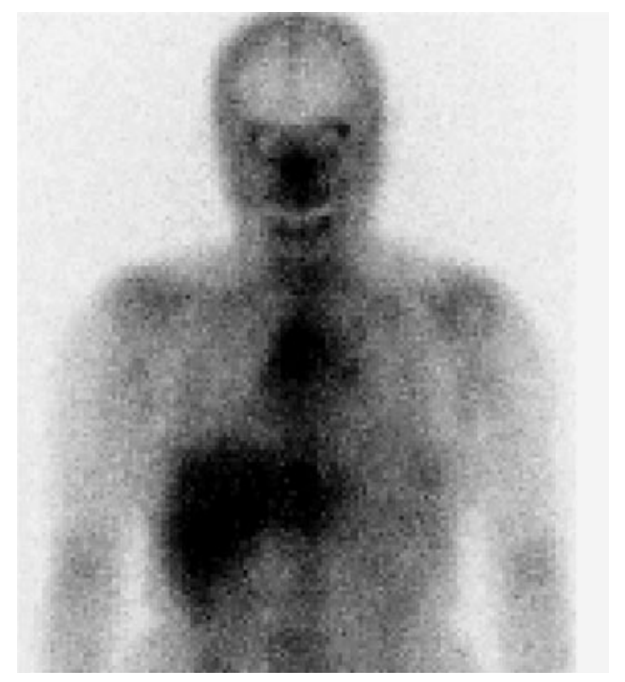

Fig. 2 Gallium-67 citrate scintigram showing intense uptake in bilateral hilar and mediastinum

Initial laboratory investigations revealed blood urea of $14 \mathrm{mg} / \mathrm{dl}$, creatinine of $0.63 \mathrm{mg} / \mathrm{dl}$ and albumin of $2.9 \mathrm{~g} / \mathrm{dl}$. Twenty-four-hour urinary protein excretion was $4.3 \mathrm{~g}$, with a selectivity index of 0.152 . Serum-corrected calcium was not elevated, and urinary $\beta 2$-microglobulin $(347 \mu \mathrm{g} / \mathrm{l})$ was not high. Nephrocalcinosis and nephrolithiasis were not detected by ultrasonography. At that time, we confirmed that sarcoid uveitis had improved and hilar lymphadenopathy had disappeared on chest CT scan.

Kidney biopsy was performed. Light microscopy revealed 36 glomeruli, none of which were globally sclerotic. Glomerular abnormalities and tubulointerstitial nephritis were not observed. Immunofluorescence microscopy showed no deposits of immunoglobulins in the glomeruli. Electron microscopy revealed diffuse foot process effacement, vacuolisation of epithelial cells and microvillous transformation. No electron-dense deposits were identified. These findings are consistent with MCD.
The patient did not use non-steroidal anti-inflammatory drugs (NSAIDs) or other medications known to cause druginduced MCD. Because MCD developed during the tapering period of prednisolone for the treatment of sarcoidosis, we used intravenous methylprednisolone (mPSL) pulse therapy $(500 \mathrm{mg} /$ day) for 3 days, followed by $40 \mathrm{mg} /$ day oral prednisolone. Twenty-six days after the initiation of mPSL pulse therapy, the patient achieved complete remission of nephrotic syndrome. Other immunosuppressive drugs were not necessary and steroids were tapered without any signs of relapse to date.

On admission we also evaluated thyroid function. Laboratory tests revealed low thyroid-stimulating hormone (TSH) $(0.36 \mu \mathrm{IU} / \mathrm{ml}$; normal, $0.50-5.00 \mu \mathrm{IU} / \mathrm{ml})$, low free triiodothyronine (FT3) $(1.26 \mathrm{pg} / \mathrm{ml}$; normal, $2.30-4.30 \mathrm{pg} / \mathrm{ml})$ and normal free thyroxine (FT4) $(1.17 \mathrm{mg} / \mathrm{dl}$; normal, $0.90-1.70 \mathrm{mg} / \mathrm{dl}$ ). Antithyroid peroxidase antibody (TPO$\mathrm{Ab})$ was normal, but antithyroglobulin antibody (Tg-Ab) was elevated ( $33 \mathrm{IU} / \mathrm{ml}$; normal, $<27 \mathrm{IU} / \mathrm{ml})$. Ultrasound showed mild enlarged thyroid gland with inhomogeneous parenchyma and hypoechoic areas. Hashimoto's thyroiditis was diagnosed. Normalisation of $\mathrm{Tg}$-Ab titres was obtained after the initiation of mPSL pulse therapy.

\section{Discussion}

Extraglomerular diseases, which are reportedly associated with MCD, are both numerous and diverse, and autoimmune thyroid disease and sarcoidosis are included in this entity [3]. A few cases of MCD in patients with Hashimoto's thyroiditis have been reported. Kagiyama et al. [4] reported a case in which MCD and Hashimoto's thyroiditis occurred in an insulin-dependent diabetes mellitus patient. Iwazu et al. [5] reported a case of simultaneous occurrence of MCD and Hashimoto's thyroiditis in the absence of any other immunologic conditions known to occur in association with MCD. Similarly, there have been at least four case reports of the concomitant occurrence of MCD and Graves' disease [6-9]. MCD has also been reported in association with sarcoidosis. Parry and Falk [10] reported a case of MCD and sarcoidosis in which MCD developed 14 years after sarcoidosis had been diagnosed. Anwar and Gokal [11] reported a case of simultaneous occurrence of sarcoidosis and $\operatorname{IgA}$ nephropathy in a patient who had previously suffered from MCD. Rarely, MCD, sarcoidosis and autoimmune thyroid disease occur in the same patients concurrently or consecutively. Mündlein et al. [12] reported a case of simultaneous occurrence of Graves' disease and sarcoidosis in a patient with MCD. The patient received a diagnosis of MCD in 1984, and Graves' disease and sarcoidosis were diagnosed in 1995. In 1996, the patient had concurrent relapses of MCD and Graves' 
disease. Nishimoto et al. [13] reported a case of concomitant occurrence of MCD, sarcoidosis and Hashimoto's thyroiditis.

As summarised above, MCD, sarcoidosis and autoimmune thyroid disease did not occur in the same order, and the disease developing first has differed from case to case. In our case, the onset of Hashimoto's thyroiditis was unclear. The patient did not have any symptoms of Hashimoto's thyroiditis and never underwent a clinical examination of thyroid function before admission. In previous reports, sarcoidosis patients, as opposed to MCD patients, had a high incidence of positive TPO-Ab and Tg-Ab [14-16]. Interestingly, many Hashimoto's thyroiditis patients with sarcoidosis have relatively low titres of $\mathrm{TPO}-\mathrm{Ab}$ and $\mathrm{Tg}-\mathrm{Ab}$ [17]. In our case, the patient was diagnosed with Hashimoto's thyroiditis, despite the low titre of Tg-Ab. This suggests that the onset of Hashimoto's thyroiditis was associated with that of sarcoidosis.

The exact mechanism underlying the pathogenesis of MCD in association with sarcoidosis and Hashimoto's thyroiditis is uncertain. It is known that the activation of $\mathrm{T}$ cells with subsequent production of various cytokines plays an important role. In our case, sarcoidosis occurred first. In sarcoidosis, in addition to the known T-helper (Th) 1 inflammation, Th17 cell inflammation and the impaired suppressive effects of regulatory $\mathrm{T}$ (Treg) cells contribute to the pathogenesis [18]. Similarly to sarcoidosis, in Hashimoto's thyroiditis, Th1 is dominant and there is increased differentiation of Th17 lymphocytes [19, 20]. On the other hand, only MCD is associated with a Th2 response [21]. Differences in Th cell balance provided further evidence that Hashimoto's thyroiditis is more strongly associated with sarcoidosis than MCD. Remarkably, glucocorticoid modulation of cytokine expression caused a shift from Th1 to Th2 immunity [22]. It is possible that steroids for the treatment of sarcoidosis improved Hashimoto's thyroiditis and induced the development of MCD. In our patient, mPSL pulse therapy and increased prednisolone dose resulted in complete remission of nephrotic syndrome. The increased Th17/Treg ratio probably returned to normal after effective steroid therapy [23].

Differences between the treatment for MCD and MCD in association with sarcoidosis and autoimmune thyroid disease are obscure. Since most of the MCD patients in association with sarcoidosis or autoimmune thyroid disease are responsive to usual steroids therapy, it is likely that sarcoidosis and autoimmune thyroid disease do not have a significant influence on the effects of steroids therapy for MCD. Similar to our case, there was a case report of MCD in a patient with sarcoidosis in which prednisolone did not work well [10]. In such situations, it was effective to increase the dose of steroids including mPSL pulse therapy or to start other immunosuppressive drugs.
To our knowledge, this is the first case report of MCD in association with sarcoidosis and Hashimoto's thyroiditis that developed despite taking moderate doses of prednisolone. The investigation of Th patterns and cytokine balance may provide further insights into the pathogenesis of MCD.

Conflict of interest The authors have declared that no conflict of interest exists.

\section{References}

1. Becker BA, Fenves AZ, Breslau NA. Membranous glomerulonephritis associated with Graves' disease. Am J Kidney Dis. 1999;33:369-73.

2. Taylor RG, Fisher C, Hoffbrand BI. Sarcoidosis and membranous glomerulonephritis: a significant association. Br Med J (Clin Res Ed). 1982;284:1297-8.

3. Glassock RJ. Secondary minimal change disease. Nephrol Dial Transplant. 2003;18(Suppl 6):vi52-8.

4. Kagiyama S, Tsuruta H, Tominaga M, Morishita K, Doi Y, Onoyama K. Minimal-change nephrotic syndrome and acute renal failure in a patient with aged onset insulin-dependent diabetes mellitus and autoimmune thyroiditis. Am J Nephrol. 1999;19:369-72.

5. Iwazu Y, Nemoto J, Okuda K, Nakazawa E, Hashimoto A, Fujio Y, Sakamoto M, Ando Y, Muto S, Kusano E. A case of minimal change nephrotic syndrome with acute renal failure complicating Hashimoto's disease. Clin Nephrol. 2008;69:47-52.

6. Hasnain W, Stillman IE, Bayliss GP. Minimal-change renal disease and Graves' disease: a case report and literature review. Nephrol Dial Transplant Plus. 2011;4:96-8.

7. Esteve Simó V, Fontseré N, Saurina A, Ramírez de Arellano M. Graves disease, hypothyroidism, and minimal-change glomerulonephritis. Nefrologia. 2008;28:230-1.

8. Holt S, Kingdon E, Morganstein D, Sweny P. Simultaneous relapse of Graves' disease and minimal change glomerular disease. Nephrol Dial Transplant. 2002;17:666-8.

9. Tanwani LK, Lohano V, Broadstone VL, Mokshagundam SP. Minimal change nephropathy and Graves' disease: report of a case and review of the literature. Endocr Pract. 2002;8:40-3.

10. Parry RG, Falk C. Minimal-change disease in association with sarcoidosis. Nephrol Dial Transplant. 1997;12:2159-60.

11. Anwar N, Gokal R. Simultaneous occurrence of IgA nephropathy and sarcoidosis in the context of pre-existent minimal change nephrotic syndrome. Nephron. 1993;65:310-2.

12. Mündlein E, Greten T, Ritz E. Graves' disease and sarcoidosis in a patient with minimal-change glomerulonephritis. Nephrol Dial Transplant. 1996;11:860-2.

13. Nishimoto A, Tomiyoshi Y, Sakemi T, Kanegae F, Nakamura M, Ikeda Y, Shimazu K, Yonemitsu N. Simultaneous occurrence of minimal change glomerular disease, sarcoidosis and Hashimoto's thyroiditis. Am J Nephrol. 2000;20:425-8.

14. Antonelli A, Fazzi P, Fallahi P, Ferrari SM, Ferrannini E. Prevalence of hypothyroidism and Graves disease in sarcoidosis. Chest. 2006;130:526-32.

15. Papadopoulos KI, Hörnblad Y, Liljebladh H, Hallengren B. High frequency of endocrine autoimmunity in patients with sarcoidosis. Eur J Endocrinol. 1996;134:331-6.

16. Isern V, Lora-Tamayo J, Capdevila O, Villabona C, Mañá J. Sarcoidosis and autoimmune thyroid disease. A case series of ten patients. Sarcoidosis Vasc Diffuse Lung Dis. 2007;24:148-52. 
17. Nakamura H, Genma R, Mikami T, Kitahara A, Natsume H, Andoh S, Nagasawa S, Nishiyama K, Chida K, Sato A, Yoshimi T. High incidence of positive autoantibodies against thyroid peroxidase and thyroglobulin in patients with sarcoidosis. Clin Endocrinol (Oxf). 1997;46:467-72.

18. Facco M, Cabrelle A, Teramo A, Olivieri V, Gnoato M, Teolato S, Ave E, Gattazzo C, Fadini GP, Calabrese F, Semenzato G, Agostini C. Sarcoidosis is a Th1/Th17 multisystem disorder. Thorax. 2011;66:144-50.

19. Phenekos C, Vryonidou A, Gritzapis AD, Baxevanis CN, Goula M, Papamichail M. Th1 and Th2 serum cytokine profiles characterize patients with Hashimoto's thyroiditis (Th1) and Graves' disease (Th2). Neuroimmunomodulation. 2004;11:209-13.
20. Figueroa-Vega N, Alfonso-Pérez M, Benedicto I, Sánchez-Madrid F, González-Amaro R, Marazuela M. Increased circulating proinflammatory cytokines and Th17 lymphocytes in Hashimoto's thyroiditis. J Clin Endocrinol Metab. 2010;95:953-62.

21. Grimbert P, Audard V, Remy P, Lang P, Sahali D. Recent approaches to the pathogenesis of minimal-change nephrotic syndrome. Nephrol Dial Transplant. 2003;18:245-8.

22. Elenkov IJ. Glucocorticoids and the Th1/Th2 balance. Ann N Y Acad Sci. 2004;1024:138-46.

23. Liu LL, Qin Y, Cai JF, Wang HY, Tao JL, Li H, Chen LM, Li MX, Li XM, Li XW. Th17/Treg imbalance in adult patients with minimal change nephrotic syndrome. Clin Immunol. 2011;139: 314-20. 